\title{
The Cost-Benefit of Federal Investment in Preventing Clostridium difficile Infections through the Use of a Multifaceted Infection Control and Antimicrobial Stewardship Program
}

\author{
Rachel B. Slayton, PhD, MPH, R. Douglas Scott II, PhD, James Baggs, PhD, Fernanda C. \\ Lessa, MD, L. Clifford McDonald, MD, and John A. Jernigan, MD \\ Division of Healthcare Quality Promotion, Centers for Disease Control and Prevention, Atlanta, \\ Georgia
}

\begin{abstract}
OBJECTIVE.-To determine the potential epidemiologic and economic value of the implementation of a multifaceted Clostridium difficile infection (CDI) control program at US acute care hospitals
\end{abstract}

DESIGN.-Markov model with a 5-year time horizon

PARTICIPANTS.-Patients whose data were used in our simulations were limited to hospitalized Medicare beneficiaries $\searrow 65$ years old.

BACKGROUND.-CDI is an important public health problem with substantial associated morbidity, mortality, and cost. Multifaceted national prevention efforts in the United Kingdom, including antimicrobial stewardship, patient isolation, hand hygiene, environmental cleaning and disinfection, and audit, resulted in a 59\% reduction in CDI cases reported from 2008 to 2012.

\begin{abstract}
METHODS.-Our analysis was conducted from the federal perspective. The intervention we modeled included the following components: antimicrobial stewardship utilizing the Antimicrobial Use and Resistance module of the National Healthcare Safety Network (NHSN), use of contact precautions, and enhanced environmental cleaning. We parameterized our model using data from CDC surveillance systems, the AHRQ Healthcare Cost and Utilization Project, and literature reviews. To address uncertainty in our parameter estimates, we conducted sensitivity analyses for intervention effectiveness and cost, expenditures by other federal partners, and discount rate. Each simulation represented a cohort of 1,000 hospitalized patients over 1,000 trials.
\end{abstract}

RESULTS.-In our base case scenario with 50\% intervention effectiveness, we estimated that 509,000 CDI cases and 82,000 CDI-attributable deaths would be prevented over a 5-year time horizon. Nationally, the cost savings across all hospitalizations would be $\$ 2.5$ billion (95\% credible interval: $\$ 1.2$ billion to $\$ 4.0$ billion).

CONCLUSIONS.-The potential benefits of a multifaceted national CDI prevention program are sizeable from the federal perspective.

Address correspondence to Rachel B. Slayton, US Centers for Disease Control and Prevention, 1600 Clifton Road, MS A-31, Atlanta, GA 30333 (via3@cdc.gov).

Potential conflicts of interest. All authors report no conflicts of interest relevant to this article. 
Clostridium difficile infection (CDI) is a substantial public health problem, particularly with the increased prevalence of the hypervirulent BI/NAP1/027 strain. ${ }^{1}$ In 2009, 336,000 CDIrelated hospital stays were reported in the United States. ${ }^{2}$ Previous studies estimated that CDI is associated with at least $\$ 3,006$ in short-term attributable costs per infection. ${ }^{3}$ The annual US burden of healthcare-associated CDI from the third-party payer perspective is at least \$547 million. ${ }^{4}$ However, few studies have estimated the economic burden of healthcare-associated infections from the federal perspective, despite the fact that the Centers for Medicare and Medicaid Services (CMS) provides federal health insurance to 44 million people $\ 65$ years old, the age group with highest prevalence of CDI. ${ }^{5}$

Between 1990 and 2004, rates of CDI in the United Kingdom dramatically increased, resulting in the Health Protection Agency and Department of Health joint update of evidence-based guidance for CDI prevention. ${ }^{6}$ This policy provided a multifaceted prevention approach including updated standardized clinical and laboratory definitions, mandatory reporting requirements, and clinical guidance for CDI management and treatment. ${ }^{6}$ Prevention strategies detailed in the core guidance in the United Kingdom focused on antimicrobial stewardship, patient isolation, hand hygiene, environmental cleaning, and a mechanism for audit. Implementation of these guidelines resulted in a 59\% reduction in CDI cases reported from fiscal year (FY) 2008 to 2012. ${ }^{7,8}$ While many of these prevention strategies have been implemented to various degrees in US hospitals, highly impactful antimicrobial stewardship appears to be the area in which US and UK hospitals differ the most. Between 2005 and 2009 there was a >40\% decline in fluoroquinolone subscribing and $>50 \%$ decline in cephalosporin prescribing in UK hospitals; meanwhile, in US hospitals, no suggestion of changes in antimicrobial prescribing has been made that might afford a similar benefit on CDI rates. ${ }^{9}$

In the United States, CDI prevention guidelines issued jointly by the Society for Healthcare Epidemiology of America (SHEA) and the Infectious Disease Society of American (IDSA) call for implementation of a multifaceted prevention strategy that closely resembles the UK approach, including antimicrobial stewardship restricting usage of antimicrobials most commonly associated with CDI. ${ }^{10}$ Examining the costs and benefits of CDI prevention in the United States, modeled after successful programs in the United Kingdom (especially more aggressive antimicrobial stewardship), would enable US federal government agencies to make evidence-based decisions about these investments.

\section{METHODS}

We constructed a Markov model using TreeAge Pro Suite 2013 (TreeAge Software, Williamstown, MA) to determine the potential epidemiologic and economic value of the implementation of a multifaceted CDI control program that includes infection control interventions and antimicrobial stewardship. We modeled the infection control portion of the intervention based upon recommendations in the SHEA/IDSA guideline, and we modeled antimicrobial stewardship as a hospital utilizing the Antimicrobial Use (AU) option of the Antimicrobial Use and Resistance (AUR) module of the National Healthcare Safety Network (NHSN) as the basis for interventions led by antimicrobial stewardship personnel. ${ }^{11}$ Standardized definitions within the AUR enable interfacility comparisons. Our analysis 
was conducted from the federal payer perspective, quantifying federal reimbursement for CDI by CMS and expenditures on CDI prevention efforts by federal agencies. We limited our study population to Medicare beneficiaries $\succ 65$ years old. We also included expenditures for infection control and stewardship efforts, as these cost increases will be included in Medicare Cost Reports and then used by CMS to adjust future reimbursement rates. The analysis was conducted over a 5-year time horizon. We created a series of models with identical structures and parameterized each model with age-stratified inputs. The model included Markov states representing a hospitalized patient's CDI-associated health status for a 1-year cycle length: (1) no CDI, (2) hospital-onset CDI (HO-CDI), community-onset hospital-associated CDI (COHA-CDI), death (absorptive). Table 1 lists the parameter estimates included in our model's base-case scenario. Each model represented a cohort of 1,000 hospitalized persons and outcomes were calculated for 1,000 trials. We determined cost per case averted, in which a case of CDI was defined as a person with either HO-CDI or COHA-CDI. We scaled our results nationally for all persons $\geq 65$ years old using agestratified estimates of hospital discharges among persons for whom Medicare was the primary payer. ${ }^{5}$

\section{Probability}

We estimated the age-stratified probability of HO-CDI using discharge data from the Healthcare Cost and Utilization Project (HCUP) for intestinal infections due to $C$. difficile (ICD-9-CM 008.45) as a secondary diagnostic code. ${ }^{2}$ To adjust for coding discrepancies (ie, ICD-9-CM codes may indicate a history of CDI rather than an incident infection ${ }^{12}$ ), we linked NY Emerging Infections Programs (EIP) CDI surveillance data (active laboratoryand population-based surveillance for $\mathrm{C}$. difficile) to administrative data from a large academic hospital participating in the NY-EIP using SAS, version 9.3 (SAS Institute, Cary, $\mathrm{NC}$ ); we then estimated the predictive value of ICD-9-CM coding for HO-CDI. ${ }^{11}$ These methods are described in detail elsewhere. ${ }^{13}$ We also computed the ratio of HO-CDI to COHA-CDI reported to NY-EIP to calibrate the probability of community-onset infections that hospital-level interventions have the potential to prevent. ${ }^{13} \mathrm{We}$ scaled our estimates from HCUP to include federal hospitals using the ratio of federal to nonfederal discharges. ${ }^{14}$ We validated our adjusted estimates of incident infections with national estimates from the EIP (unpublished data). To remain conservative about the potential benefits of the intervention and to account for increased sensitivity of nucleic acid amplification testing (NAAT) on population-based incidence, we assumed that in the absence of intervention, incidence remained at 2011 levels. Age-specific probability of non-CDI-attributable mortality was estimated from the Human Mortality Database. ${ }^{15}$

\section{CDI-Associated Costs}

To determine the attributable excess reimbursement made by CMS as a third-party payer for persons with HO-CDI, we linked Medicare Provider Analysis and Review (MEDPAR) ${ }^{16}$ and NHSN data. MEDPAR includes individual-level claims for services provided to Medicare beneficiaries at certified inpatient hospitals and skilled nursing facilities. To estimate the excess attributable reimbursement and length of stay, we identified hospitalizations among Medicare recipients during which an episode of HO-CDI occurred, then we compared those hospitalizations to admissions among a similar population of Medicare recipients who did 
not develop CDI. Frequency matching and multivariate median regression controlled for potential confounders, including receipt of ICU care, facility characteristics, demographics, clinical factors, comorbidity score, and primary ICD9-CM procedure category based on AHRQ Clinical Classification Software. ${ }^{17,18}$ We also determined the additional reimbursements attributable to hospitalization for recurrent CDI within 8 weeks of the index hospitalization-discharge. To remain conservative, we only included attributable reimbursement for the first hospitalization for recurrence. For cases of COHA-CDI, we used the CMS Chronic Condition Data Warehouse to determine the reimbursement for hospitalization due to CDI (having a primary ICD-9-CM code for CDI), within 8 weeks of an index non-CDI hospitalization. ${ }^{19} \mathrm{We}$ attributed the entire cost of such hospitalizations to CDI assuming that those hospitalizations would not have occurred but for COHA-CDI. We determined the additional attributable reimbursement for the first hospitalization for recurrent CDI occurring within 8 weeks COHA-CDI discharge. Past costs were converted to 2011 US dollars using the Consumer Price Index for Urban Consumers (CPI-U) for medical care. A $3 \%$ discount rate accounted for time preference. ${ }^{20}$

\section{Intervention Components}

We based the potential effectiveness of CDI control programs on the experience documented in the United Kingdom, where a multifaceted infection control and antimicrobial stewardship was implemented nationally and resulted in a 59\% reduction on the number of CDI cases reported from fiscal year 2008 to 2012.7,8 Our base-case scenario evaluated an intervention effectiveness of $50 \%$, where we assumed that a $50 \%$ reduction in first year would be sustained over the 5-year program. We estimated the hospital cost per discharge of implementing the AU module and the ongoing cost of maintenance by tabulating personnel time by occupational category and equipment costs using key informant interviews at NHSN AU module pilot sites (ie, facilities that were early adopters of the AU module and provided feedback for continued development). To account for time for antimicrobial stewardship activities, we estimated personnel time dedicated to stewardship from the published literature and category-specific wages from the Bureau of Labor Statistics. ${ }^{21-23}$ These costs were evenly attributed across all discharges, regardless of CDI status. To remain conservative about the potential benefits, we assumed that all patients with CDI would be placed on contact precautions for the length of their hospitalization, which would tend to overestimate the cost of isolation because current SHEA/IDSA guidelines recommend contact precautions only for the duration of diarrhea. ${ }^{10}$ We modeled the cost of contact precautions as the number of patient contacts per day multiplied by the cost of gloves and gown and 1 minute of nurse time for donning and doffing for the duration of hospitalization. Finally, we modeled improved environmental cleaning as the change from liquid quaternary ammonium disinfectant to a 1:10 dilution of sodium hypochlorite solution (eg, bleach registered with the US Environmental Protection Agency as having efficacy against CDI). ${ }^{22}$ Our cleaning cost estimate included 2 hours of cleaning personnel supervisor time per week for audit and feedback per hospital.

We quantified the Centers for Disease Control and Prevention (CDC) expenditures on CDI prevention and control by reviewing $\mathrm{CDC}$ annual program budgets from the Office of Chief Financial Officer. CDC expenditures on CDI prevention encompassed disease surveillance, 
research studies, and outbreak investigation; this included dedicated personnel time, laboratory supplies and contracts, extramural funding, and development and support of NHSN modules. We stratified these costs into initial investments (ie, occurring through 2011) and ongoing investments (ie, actual annual expenditures in 2012 and projected expenditures for fature years).

\section{Sensitivity Analyses}

To address uncertainty in key model parameters, we conducted sensitivity analyses systematically varying parameters to determine their impact. Data regarding the effectiveness of preventing CDI are largely based on estimates from the United Kingdom or single US hospital studies and may have limited generalizability. $7,21,23,24$ Therefore, we varied the effectiveness of the intervention from $10 \%$ to $75 \%$. We also varied the percentage of stewardship costs that are attributable to CDI prevention activities from $25 \%$ (base case) to $100 \%$. To evaluate the impact of expenditures by other federal partners (eg, NIH, AHRQ, etc.), we performed a sensitivity analysis of expenditures by other federal partners relative to the total CDC expenditures per discharge from 2 to 10 times the initial and ongoing CDC expenditures. Finally, we systematically varied the discount rate used from $0 \%$ to $3 \%$ (base case).

\section{RESULTS}

In our base-case scenario, in which intervention effectiveness was assumed to be $50 \%$, we estimated that $36.94 \mathrm{CDI}$ cases requiring admission could be averted per 1,000 persons $\searrow 65$ years old over a 5-year time horizon, comprising 15.46 cases of HO-CDI and 21.48 hospitalizations due to COHA-CDI averted. Benefits peaked in persons 70-79 years old, in whom the risk of CDI is greatest but the mortality from other causes is relatively low (Table 2). Additionally, $5.91 \mathrm{CDI}$-attributable deaths per 1,000 would be averted over 5 years: 2.43 deaths among persons with HO-CDI and an additional 3.48 deaths among persons with COHA-CDI. This estimate equates to a cumulative 509,000 CDI cases and 82,000 CDIattributable deaths over 5 years. The mean cost-savings per each hospitalization among Medicare beneficiaries was estimated to be $\$ 184$ (95\% credible interval: $\$ 90$ to $\$ 287$ ) over 5 years. Nationally, the cost savings across all hospitalizations would be $\$ 2.5$ billion (95\% credible interval: $\$ 1.2$ billion to $\$ 4.0$ billion) over 5 years.

Table 2 outlines the number of incident CDI cases we estimated would be averted by varying intervention effectiveness. Figure 1 delineates the cumulative modeled cost-benefit by intervention effectiveness over our study's 5-year time horizon for a cohort of persons 65-69 years old. In general, interventions with at least moderate effectiveness (ie, $25 \%$ ) would be cost saving in older populations (Figure 1). Cost-benefit increases as intervention effectiveness increases. When intervention effectiveness was set to $10 \%$, the model predicted that an estimated 101,000 cases and 16,000 CDI-attributable deaths would be averted nationally over 5 years. When intervention effectiveness was assumed to be $75 \%$, an estimated 773,000 cases and 124,000 CDI-attributable deaths would be averted nationally over 5 years. 
Our model was moderately sensitive to our assumption of the proportion of hospital costs of implementing a multifaceted intervention including antimicrobial stewardship. For example, our mean estimate of the economic value of implementation was no longer cost saving when we attributed 50\% of hospital-level stewardship and infection control costs to CDI prevention and program effectiveness was $<10 \%$. Attributing $50 \%$ of stewardship program costs to CDI prevention of a program with $40 \%$ effectiveness, we estimate that among a cohort of persons 65-69 years old, the cost savings would be \$124 per hospitalization (95\% credible interval: \$42-\$214). When we attributed $100 \%$ of stewardship personnel costs to CDI prevention, our mean estimate of economic value was no longer cost saving when program effectiveness was $<15 \%$. The economic value of a program with $100 \%$ of stewardship personnel costs attributed to CDI and 10\% program effectiveness had an estimated economic value of $-\$ 9$ per hospitalization (95\% credible interval: $-\$ 52$ to $\$ 50$ ).

We independently evaluated the impact of expenditures by other federal agencies on the economic value of a multifaceted intervention, including antimicrobial stewardship, returning to our base-case scenario for all other parameters. When these expenditures were assumed to be 10 times CDC expenditures (ie, other federal expenditures were initially $\approx$ $\$ 108$ million and $\approx \$ 34$ million ongoing), we estimated that the economic value of a multifaceted intervention, including antimicrobial stewardship with $50 \%$ effectiveness for a cohort of 1,000 hospitalized person 65-69 years old, would be \$170 per hospitalization (95\% credible interval: \$83-\$264). Our economic value estimate did not change substantially when we varied the discount rate from $0 \%$ to $3 \%$.

\section{DISCUSSION}

Our study highlights the sizable potential benefits of federal investment in a multifaceted national CDI prevention program. Our analysis suggests CDI prevention is cost saving, and we estimated that the cumulative economic benefit in reimbursements for the Medicare and Medicaid programs over 5 years would be $\$ 2.5$ billion (95\% credible interval: $\$ 1.2$ billion to $\$ 4.0$ billion) with 509,000 CDI cases averted nationally.

We evaluated the impact of a multifaceted CDI prevention program based on best practices in the current literature. While we can estimate the cumulative effectiveness of such a program, there are insufficient data to estimate the incremental contribution of each component in a multifaceted intervention. However, evidence suggests that antimicrobial stewardship may have been a key driver of the impressive progress in CDI prevention in the United Kingdom. ${ }^{7,9}$ Disturbance of the intestinal microbiota from exposure to antimicrobial agents is a significant modifiable risk factor for CDI acquisition. ${ }^{25}$ A systematic review that included 3 interrupted time series analyses conducted in the United States measuring the impact of antimicrobial stewardship on CDI colonization or infection concluded that, 1 year after implementation, CDI reduction ranged from $52 \%$ to $79 \% .{ }^{26}$ This finding suggests that the success of prevention efforts in the United Kingdom is not isolated but rather that it is a credible goal for US prevention programs. In addition to improving routine patient care, antimicrobial stewardship has also been a key provision of CDI outbreak mitigation, notably during a recent Canadian outbreak. ${ }^{27}$ 
Given the body of evidence supporting antimicrobial stewardship for CDI prevention, major efforts to ensure widespread implementation of antimicrobial stewardship are warranted. Indeed, the importance of a multifaceted infection prevention strategy including antimicrobial stewardship as a mechanism to reduce the occurrence of multidrug-resistant infections and to safeguard limited treatment options, was reiterated in a 2012 joint policy statement by SHEA, IDSA, and the Pediatric Infectious Disease Society (PIDS) calling on the CMS to "improve the development, implementation, and monitoring of antimicrobial stewardship plans and programs over time by requiring additional activities." 28 A critical component of antimicrobial stewardship is standardized measurement of the quantity of antiinfective medications used in facilities and identification of opportunities for improvement through systems such as the NHSN AUR module.

Our analysis was subject to several limitations. First, we used administrative data adjusted with EIP analyses to calibrate our model's probability of CDI. These data were from one EIP site and may not be generalizable; however, these adjustments to ICD-9-CM coded diagnoses were consistent with a single hospital study and our model's estimates of CDI burden were validated with national EIP estimates. ${ }^{15} \mathrm{CMS}$ implemented an incentive in January 2013 for acute care hospitals to report CDI to NHSN and has proposed that longterm acute care hospitals also report beginning in FY2017. ${ }^{29}$ These data could be used in the future to recalibrate our model and generate more precise estimates of projected burden and benefit. Moreover, these data, linked with Medicare Part B (ie, physician reimbursement) and Medicare Part D (ie, drug reimbursement) data could be used to capture Medicare reimbursements resulting from hospital-associated CDI that occurs post-discharge in nursing home or outpatient settings not resulting in readmission. This procedure would likely improve the economic value of acute-care hospital based antimicrobial stewardship since our current analysis would be an underestimate. Second, our estimates of personnel required to implement a stewardship program were based on published studies from large academic medical centers and may be different in smaller non-academic medical centers. We used estimates from large stewardship programs, which are likely to have higher costs to remain conservative about the potential benefits of a stewardship program. Our model was moderately sensitive to this parameter; therefore having a better estimate of costs associated with antimicrobial stewardship across hospital types would be valuable. Additionally, if a subset of facilities has already implemented these interventions independently, the economic value would be toward the lower end of our sensitivity analysis estimates, but would still represent a substantial savings.

Uncertainty remains for some model parameters, including multifaceted intervention effectiveness in US hospitals; however our findings were robust across a wide range of effectiveness values. Even when assuming the intervention's effectiveness to be half that in the United Kingdom, cost savings were still demonstrated. In additional sensitivity analyses, the intervention remained cost saving, even with more modest reductions in CDI during the initial year with continued reductions in subsequent years (results not shown). As data become available, we can recalibrate our model with cost estimates of stewardship and demonstrated effectiveness of these programs across hospital types to assess economic value. In this analysis we endeavored to maximize program costs and to remain conservative in our overall estimate of economic value. Our study highlights the sizable potential benefits 
of multifaceted prevention programs that include the critical component of antimicrobial stewardship through use of the NHSN's AUR module for the prevention of CDI among older adults.

\title{
ACKNOWLEDGMENTS
}

\author{
We acknowledge the assistance of Ghinwa Dumyati, MD, and Elizabeth Dodds Ashley, PharmD, from the \\ University of Rochester, Rochester, New York.
}

Financial support: No financial support was provided relevant to this article.

DISCLAIMER

The findings and conclusions in this report are those of the authors and do not necessarily represent the official position of the Centers for Disease Control and Prevention.

\section{REFERENCES}

1. Lessa FC, Gould CV, McDonald LC. Current status of Clostridium difficile infection epidemiology. Clin Infect Dis 2012;55:S65-S70. [PubMed: 22752867]

2. Lucado J, Gould C, Elixhauser A. Clostridium difficile infections (CDI) in hospital stays, 2009: Statistical Brief \#124 Healthcare Cost and Utilization Project (HCUP) Statistical Briefs Rockville, MD: Agency for Health Care Policy and Research, 2006.

3. Dubberke ER, Olsen MA. Burden of Clostridium difficile on the healthcare system. Clin Infect Dis 2012;55(Suppl 2):S88-S92. [PubMed: 22752870]

4. McGlone SM, Bailey RR, Zimmer SM, et al. The economic burden of Clostridium difficile. Clin Microbiol Infect 2012;18:282-289. [PubMed: 21668576]

5. Bradley T, Topoleski J. An overview of the medicare program Congressional Budget office website. http://www.cbo.gov/publication/44587. Published 2013. Accessed May 2014.

6. Clostridium difficile infection: how to deal with the problem. Public Health England website. http:// www.hpa.org.uk/webc/HPAwebFile/HPAweb_C/1232006607827. Published 2008. Accessed October 23, 2013.

7. Summary points on Clostridium difficile Infection (CDI). Public Health England website. http:// www.hpa.org.uk/webc/HPAwebFile/HPAweb_C/1278944283388. Published 2013. Accessed October 9, 2013.

8. Operational guidance for HPUs on HCAI in health/social care. Public Health England website. http://www.hpa.org.uk/hpr/archives/2012/hpr2912.pdf. Published 2012. Accessed October 9, 2013.

9. Ashiru-Oredope D, Sharland M, Charani E, McNulty C, Cooke J, Group AAS. Improving the quality of antibiotic prescribing in the NHS by developing a new antimicrobial stewardship programme: start smart—then focus. J Antimicrob Chemotherapy 2012;67: i51-i63.

10. Cohen SH, Gerding DN, Johnson S, et al. Clinical practice guidelines for Clostridium difficile infection in adults: 2010 update by the society for healthcare epidemiology of America (SHEA) and the infectious diseases society of America (IDSA). Infect Control Hosp Epidemiol 2010;31:431-455. [PubMed: 20307191]

11. Antimicrobial Use and Resistance (AUR) module. Centers for Disease Control and Prevention website. http://www.cdc.gov/nhsn/PDFs/pscManual/11pscAURcurrent.pdf. Published 2013. Accessed September 25, 2013.

12. Dubberke ER, Butler AM, Nyazee HA, et al. The impact of ICD-9-CM code rank order on the estimated prevalence of Clostridium difficile infections. Clin Infect Dis 2011;53:20-25. [PubMed: 21653298]

13. Baggs J, Yousey-Hindes K, Dodds-Ashley E, et al. Identification of "high" risk population for future Clostridium difficile infection (CDI) paper presented at IDWeek2013; October 2-6, 2013, San Francisco, California. 
14. Annual survey database. American Hospital Association website. http://www.ahadata.com/ahadata/ html/AHASurvey.html. Published 2012. Accessed September 25, 2013.

15. Emerging infections program: healthcare associated infections project. Centers for Disease Control and Prevention website. http://www.cdc.gov/hai/eip/clostridium-difficile.html. Published 2012. Accessed September 30, 2013.

16. CMS Medicare Provider Analysis and Review (MEDPAR) file, 2008 and 2009. Centers for Medicare \& Medicaid Services website. http://www.cms.gov/Research-Statistics-Data-andSystems/Statistics-Trends-and-Reports/MedicareFeeforSvcPartsAB/MEDPAR.html. Published 2011. Accessed June 2013.

17. Yi SH, Baggs J, Gould CV, Scott RD 2nd, Jernigan JA Medicare reimbursement attributable to catheter-associated urinary tract infection in the inpatient setting: a retrospective cohort analysis. Med Care 2014;52:469-478. [PubMed: 24699236]

18. Malpiedi PJ, Peterson KD, Soe MM. 2011 National and state healthcare-associated infection standardization infection ratio report Atlanta, GA: Centers for Disease Control and Prevention, 2013.

19. Chronic Condition Data Warehouse website. https://www.ccwdata.org/web/guest/home. Published 2013. Accessed September 25, 2013.

20. Archived Consumer Price Index Detailed Report Information. Bureau of Labor Statistics website. http://www.bls.gov/cpi/cpi_dr.htm\#2012. Published 2013. Accessed May 2014.

21. Palmer HR, Weston J, Gentry L, et al. Improving patient care through implementation of an antimicrobial stewardship program. AJHP 2011;68:2170-2174. [PubMed: 22058103]

22. Beardsley JR, Williamson JC, Johnson JW, Luther VP, Wrenn RH, Ohl CC. Show me the money: long-term financial impact of an antimicrobial stewardship program. Infect Control Hosp Epidemiol 2012;33:398-400. [PubMed: 22418636]

23. Standiford HC, Chan S, Tripoli M, Weekes E, Forrest GN. Antimicrobial stewardship at a large tertiary care academic medical center: cost analysis before, during, and after a 7-year program. Infect Control Hosp Epidemiol 2012;33:338-345. [PubMed: 22418628]

24. Sick AC, Lehmann CU, Tamma PD, Lee CK, Agwu AL. Sustained savings from a longitudinal cost analysis of an internet-based preapproval antimicrobial stewardship program. Infect Control Hosp Epidemiol 2013;34:573-580. [PubMed: 23651887]

25. Owens RC Jr., Donskey CJ, Gaynes RP, Loo VG, Muto CA Antimicrobial-associated risk factors for Clostridium difficile infection. Clin Infect Dis 2008;46(Suppl 1):S19-S31. [PubMed: 18177218]

26. Davey P, Brown E, Charani E, et al. Interventions to improve antibiotic prescribing practices for hospital inpatients. Cochrane Database Syst Revs 2013;4:CD003543.

27. Valiquette L, Cossette B, Garant MP, Diab H, Pepin J. Impact of a reduction in the use of high-risk antibiotics on the course of an epidemic of Clostridium difficile-associated disease caused by the hypervirulent NAP1/027 strain. Clin Infect Dis 2007;45:S112-S121. [PubMed: 17683015]

28. Society for Healthcare Epidemiology of America; Infectious Diseases Society of America; Pediatric Infectious Diseases Society. Policy statement on antimicrobial stewardship by the Society for Healthcare Epidemiology of America (SHEA), the Infectious Diseases Society of America (IDSA), and the Pediatric Infectious Diseases Society (PIDS). Infect Control Hosp Epidemiol 2012;33:322-327. [PubMed: 22418625]

29. Fact sheets: CMS proposals to improve quality of care during hospital inpatient stays. Centers for Medicare and Medicaid Services website http://www.cms.gov/Newsroom/MediaReleaseDatabase/ Fact-Sheets/2013-Fact-Sheets-Items/2013-04-262.html. Published 2013. Accessed October 17, 2013.

30. Overview of the Nationwide Inpatient Sample (NIS). Healthcare Cost and Utilization Project (HCUP) website. www.hcup-us.ahrq.gov/nisoverview.jsp. Published 2013. Accessed September $25,3013$.

31. Sievert DM, Ricks P, Edwards JR, et al. Antimicrobial-resistant pathogens associated with healthcare-associated infections: summary of data reported to the National Healthcare Safety Network at the Centers for Disease Control and Prevention, 2009-2010. Infect Control Hosp Epidemiol 2013;34:1-14. [PubMed: 23221186] 
32. Jernigan JA, Clemence MA, Stott GA, et al. Control of methicillin-resistant Staphylococcus aureus at a university hospital: one decade later. Infect Control Hosp Epidemiol 1995;16:686-696. [PubMed: 8683086]

33. Puzniak LA, Gillespie KN, Leet T, Kollef M, Mundy LM. A cost-benefit analysis of gown use in controlling vancomycin-resistant Enterococcus transmission: is it worth the price? Infect Control Hosp Epidemiol 2004;25:418-424. [PubMed: 15188849]

34. May 2011 National Occupational Employment and Wage Estimates United States. Bureau of Labor Statistics website www.bls.gov/oes/current/oes_nat.htm. Published 2013. Accessed September 25, 2013.

35. Clean Quick Liquid Quaternary Sanitizer. Janitor's World website. www.janitors-world.com/ clquliqusa.html. Published 2013. Accessed October 28, 2013.

36. Dispatch Hospital Cleaner Disinfectants with Bleach. Staples website. http://www.staples.com/ Dispatch-Hospital-Cleaner-Disinfectants-with-Bleach-128-oz-Refill-4-Pack/product_109677. Published 2013. Accessed October 28, 2013. 


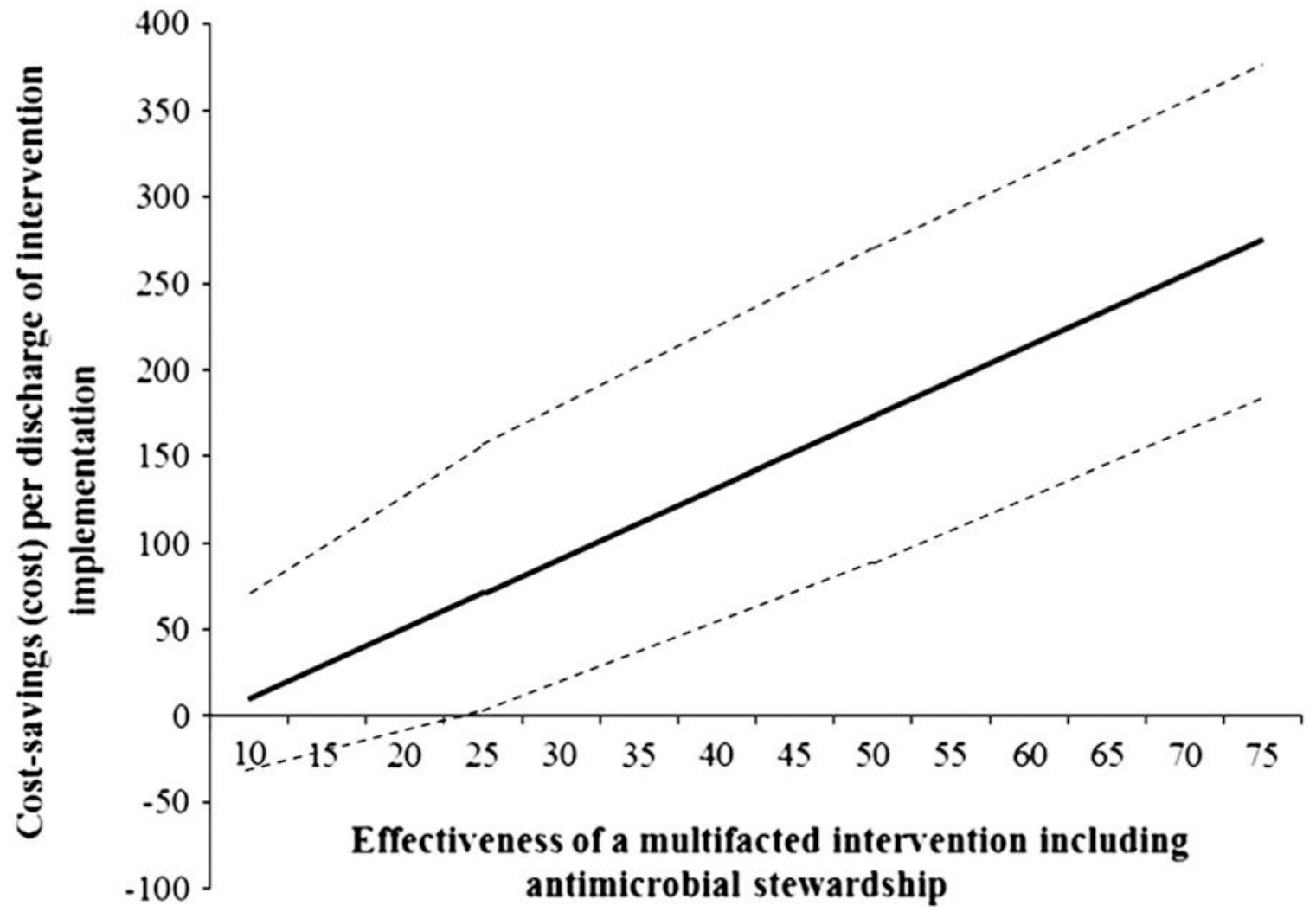

FIGURE 1.

This figure depicts the model predicted incremental (per discharge) cost savings of implementing and maintaining a multifaceted prevention program including antimicrobial stewardship program over a 5-year time horizon for a cohort of 1,000 persons aged 65-69. The solid dark line represents the mean economic value and the dotted lines represent the 95\% credible interval. Positive values indicate cost savings (ie, scenarios in which implementing the intervention is less costly than the comparator of no intervention) and negative values indicate additional expenditures with the intervention. 


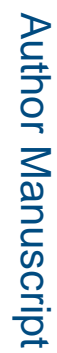

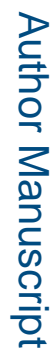

要

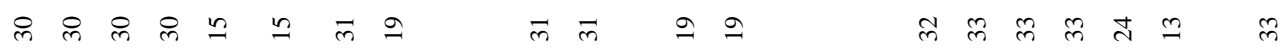

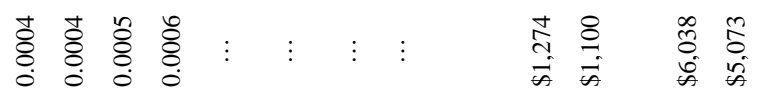

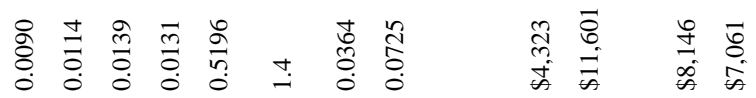$$
\infty \begin{gathered}
0 \\
\dot{\leftrightarrow}
\end{gathered}
$$
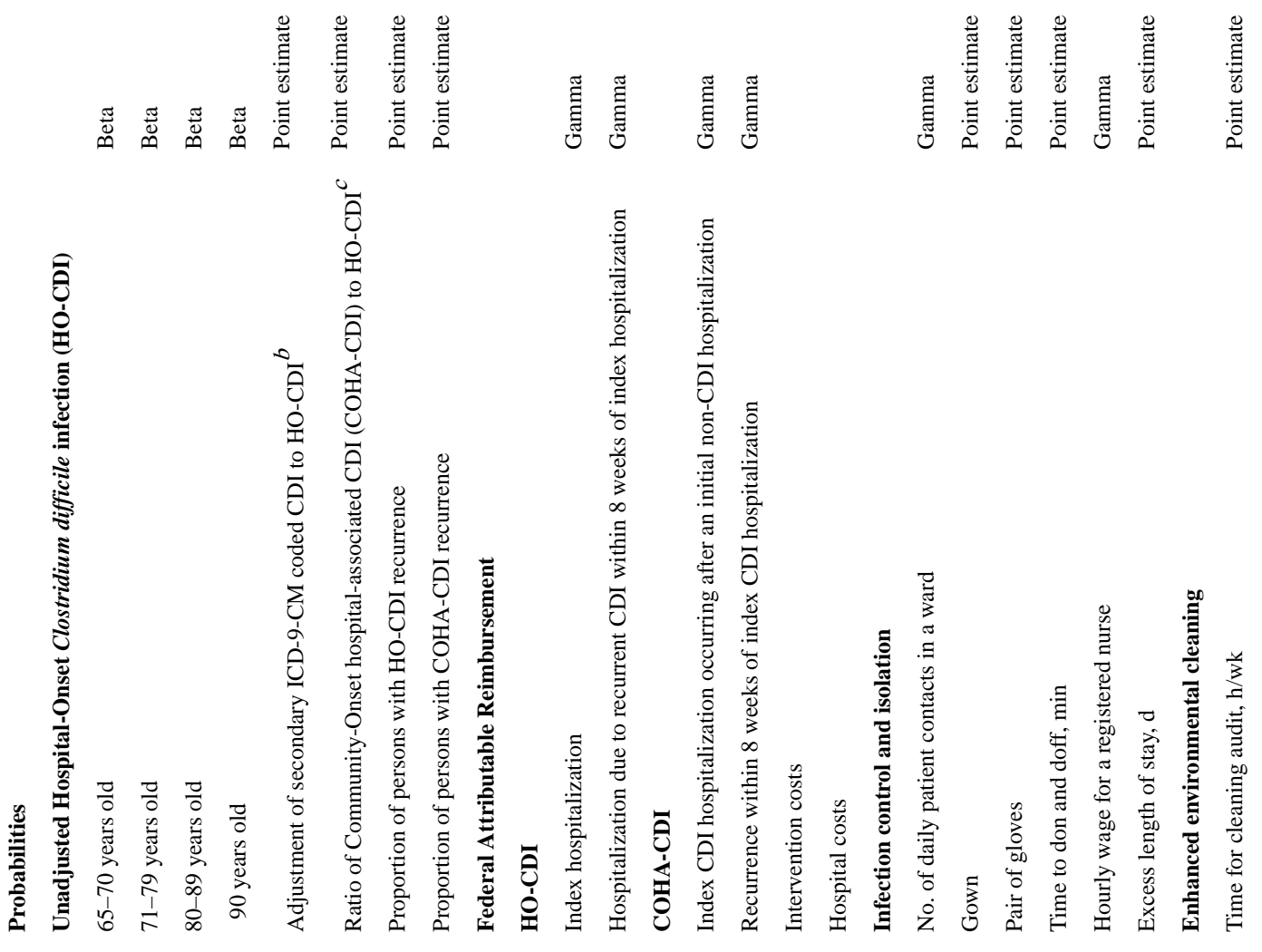


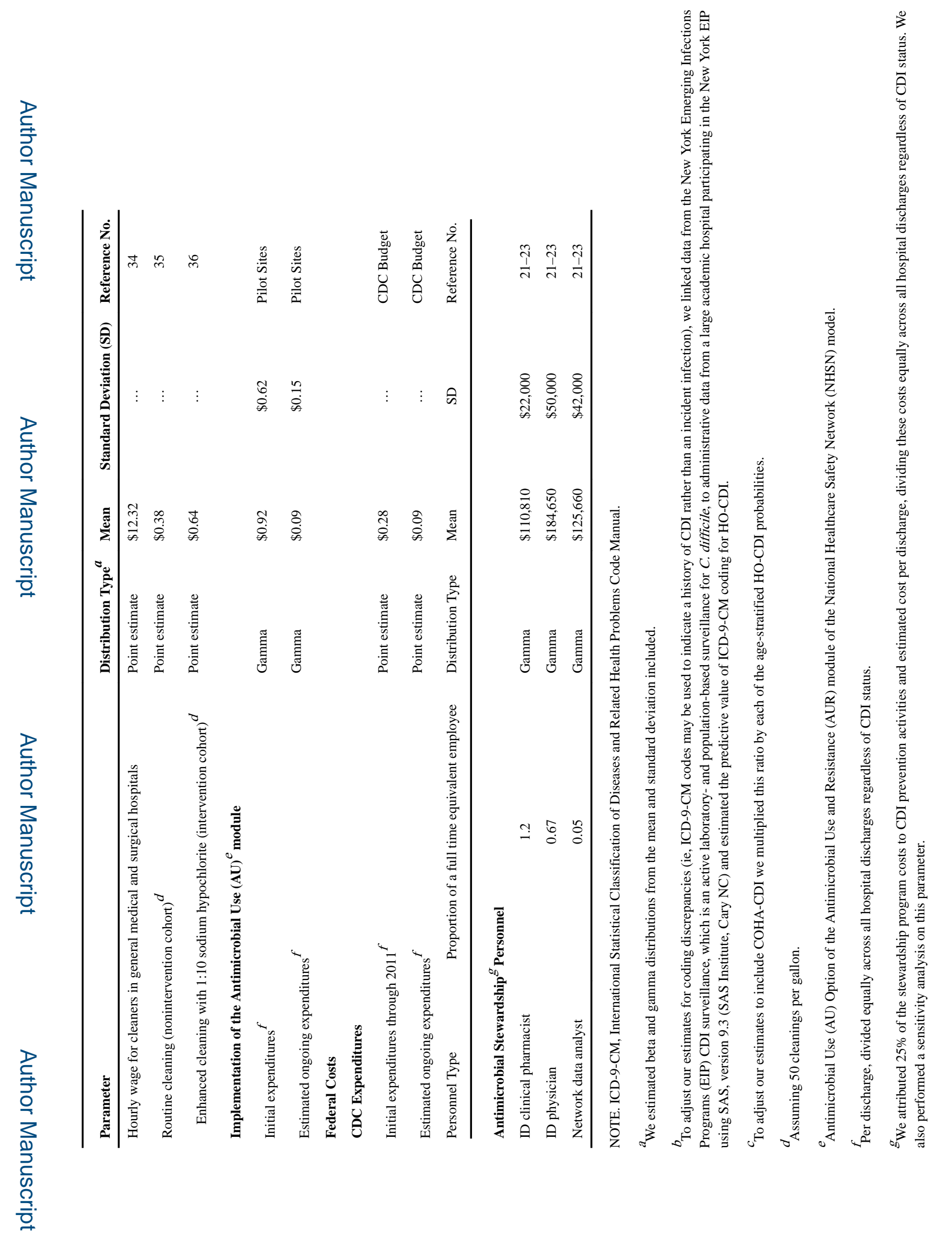

Infect Control Hosp Epidemiol. Author manuscript; available in PMC 2019 June 05. 


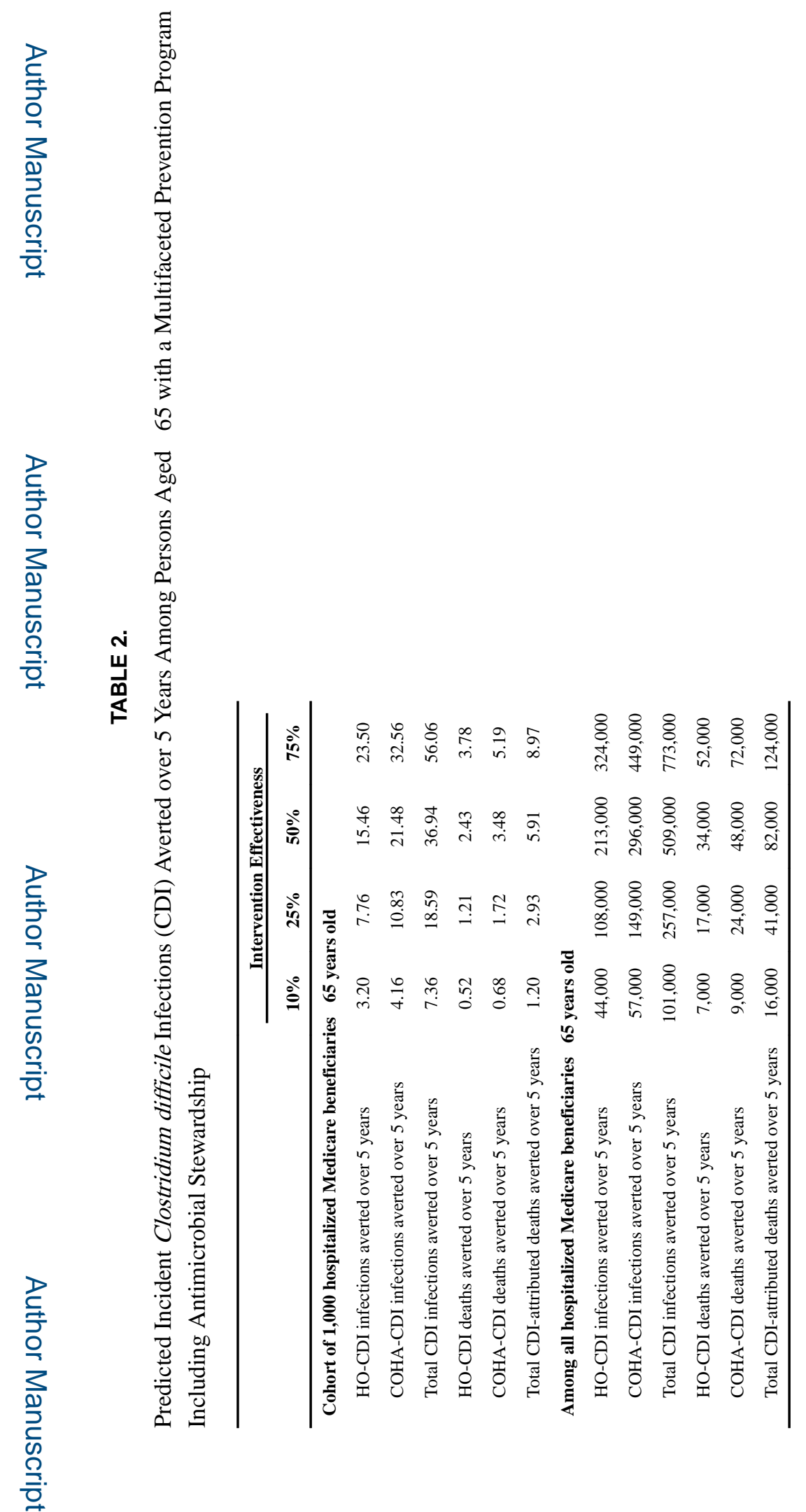

Infect Control Hosp Epidemiol. Author manuscript; available in PMC 2019 June 05. 